\title{
Assessment of Sequence Extraction Methods Applied to MMC- SDBC STATCOM Under Distorted Grid Conditions
}

This paper was downloaded from TechRxiv (https://www.techrxiv.org).

\section{LICENSE}

CC BY 4.0

SUBMISSION DATE / POSTED DATE

$05-11-2021 / 09-11-2021$

\section{CITATION}

QORIA, Taoufik; Meligy, Ahmed; Colak, Ilknur (2021): Assessment of Sequence Extraction Methods Applied to MMC-SDBC STATCOM Under Distorted Grid Conditions. TechRxiv. Preprint.

https://doi.org/10.36227/techrxiv.16941298.v1

$\mathrm{DOI}$

10.36227/techrxiv.16941298.v1 


\title{
Assessment of Sequence Extraction Methods Applied to MMC-SDBC STATCOM Under Distorted Grid Conditions
}

\author{
Ahmed Meligy, Taoufik Qoria, Ilknur Colak, Senior Member, IEEE
}

\begin{abstract}
Under grid distortions, Modular Multilevel Converters (MMC) must adopt proper control strategies to fulfill the power system requirements and ensure a stable operation. An inappropriate control under such conditions may lead to energy unbalances between the MMC legs, inaccurate current injection, and failure in the synchronization process. In this context, sequence extraction methods play a critical role in enhancing the performance of the control, firstly, by aiding the PhaseLocked Loop (PLL) to maintain the synchronization with the AC grid by following the positive sequence fundamental component of the voltage, secondly, by allowing an accurate active and reactive currents injection via the decoupled Voltage Oriented Control (dVOC), thirdly, by properly managing the internal energy of the MMC through the circulating current control. In prior researches, some sequence extraction methods have been used for MMC STATCOM. However, the sequence extraction was not the core of the performed studies and their impact on the system behavior has not been highlighted or tested in several grid conditions. This work fills this gap by first assessing the performance of a Single Delta Bridge Cell MMC (SDBC-MMC) STATCOM with four well-known sequence extraction methods (i.e., Decoupled Double Synchronous Reference Frame (DDSRF), Dual Second Order Generalized Integrator (DSOGI), Improved DSOGI, and Fortescue matrix-based (FMB) filter) under normal and abnormal grid conditions, then, finding the most suitable one in terms of stability, dynamics, and functionalities.
\end{abstract}

Index Terms-SBDC-MMC, sequence extraction methods, Harmonics, Unbalanced Grid Voltage, DC voltage offset.

\section{INTRODUCTION}

D UE to the increase of non-linear and unbalanced loads, in the modern medium voltage (MV) grids, concerns about power quality are on the rise [1]. To mitigate these issues, STATCOM units are used to maintain appropriate performances under distorted grid conditions while respecting the grid code requirements [2]. Different MMC-based STATCOM topologies have been discussed in the literature for MV applications [3]: Single Star Bridge Cell (SSBC), Single Delta Bridge Cell (SDBC), Double Star Chopper Cell (DSCC), and Double Star Bridge Cell (DSBC). Referring to [4], the SDBC MMC, also named Delta Configured Cascaded $\mathrm{H}$-Bridge converter (CHB) [5] is the most suitable MMC topology for STATCOM applications. Besides its high scalability, modularity, and efficiency, the SDBC has the advantage over the SSBC for its capability to regulate both positive and negative sequences of the reactive power and to ensure

Ahmed Meligy, Taoufik Qoria and Ilknur Colak are with Maschinenfabrik Reinhausen, Medium Voltage Power Electronics R\&D Department, Regensburg, 93059 Germany e-mail: (t.qoria@ reinhausen.com). capacitor voltage balancing thanks to the circulating current flowing through its legs [3]. Additionally, from an economic point of view, the SDBC is more convenient as it requires less full-bridge cells compared to the double star topology [3].

For STATCOM applications, it is crucial to have a reliable operation and to ensure system stability during grid distortions caused by several factors e.g., DC offsets [6], [7], harmonics [8], and voltage unbalances [9]. DC components in the AC grid are mainly due to voltage or current measurements deviation, switching devices being non-linear, and A/D conversion units [6], [10]. DC offsets along with harmonics that come from non-linear loads may engender unwanted oscillations that affect the control operation [7] and lead to the malfunction of the overall system. On the other hand, the grid voltage unbalance resulting from single or bi-phase faults [11] gives rise to a negative sequence component that in turn results in the appearance of $2 \omega$ oscillations caused by the coupling between the positive and negative sequences [8], [12]-[14]. These oscillations affect the grid voltage and current, and consequently lead to an inaccurate estimation of the grid angle and frequency, a fluctuation of the injected reactive power, and an unbalance of the energy between the MMC legs [5]. To mitigate these undesirable distortions, appropriate control strategies should be utilized, where the sequence extraction method plays an important role [15], [16]. It provides each symmetrical component separately and thereby enables the STATCOM to independently control the positive and negative sequence of the current and the voltage. In other words: 1) it allows the PLL to track the positive component of the AC voltage, and thus estimate properly the grid frequency and angle, 2) it decouples the grid current controller into positive and negative sequence current loops, and therefore gives more degrees of freedom to the control, 3) it allows the calculation of the required amount of the active power that serves to balance the clusters' voltages. Many research have been devoted to investigate the control of the SDBCMMC STATCOM under unbalanced conditions, and which incorporates a sequence extraction method. Nevertheless, these prior works either do not specify the used extraction technique, or they do not justify the reason for their choice. Authors in [9] and [17] propose a cluster voltage balancing technique for SDBC and SSBC, and test different degrees of load unbalance on both topologies. Although sequence extraction methods were implemented in these works, the exact method was not specified. Similarly, the authors in [18] propose a control to limit the peak current of the zero sequence to balance the 


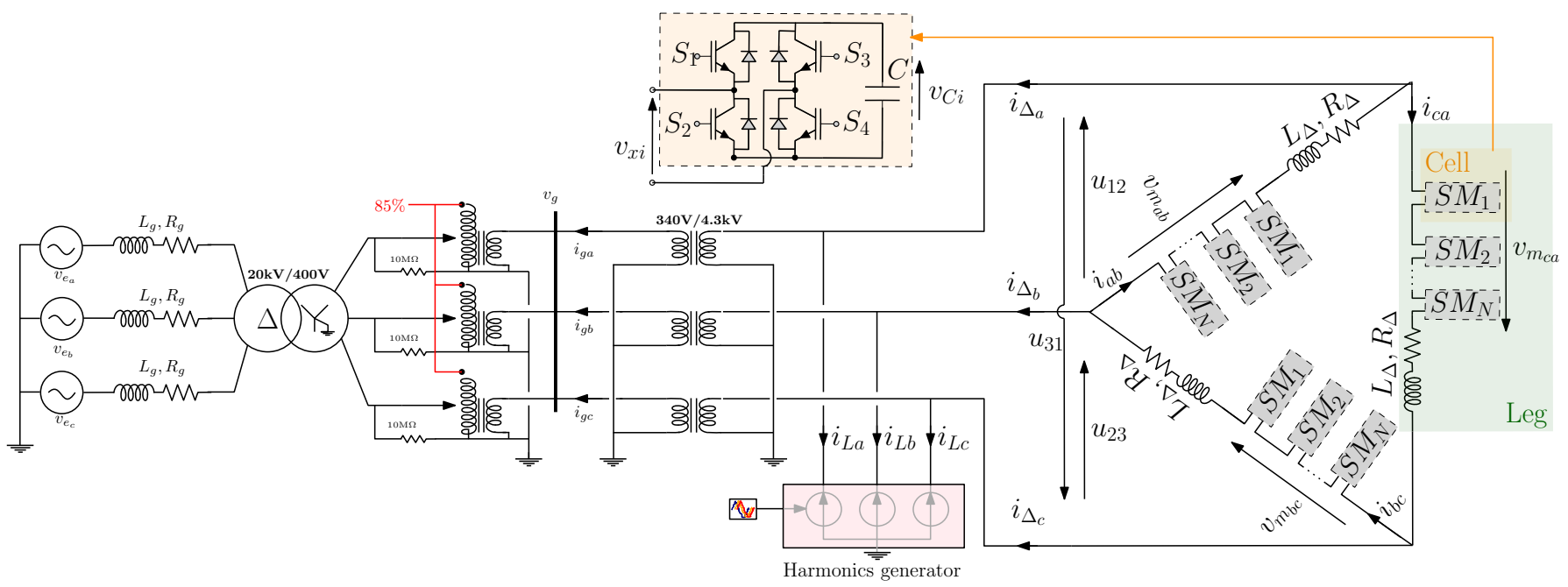

Fig. 1. Model of MMC SDBC STATCOM Connected to 20kV distribution power system through Maschinenfabrik Reinhausen platform

DC voltage. This technique also uses a sequence extraction method, nevertheless, the technique has not been specified. In [5], the authors implement a current limitation technique for SDBC STATCOM and compare the fault-ride through capabilities under balanced and unbalanced conditions. Sequence extraction is incorporated into the control, but the method was not specified. Authors in [19] propose a feedforward control method that could improve the dynamics of the circulating current control in unbalanced conditions. However, no specification on the extraction method was given in this work. On the contrary, the study conducted by [20], which compares the capability of SDBC and SSBC topologies to inject negative sequence current utilizes the Delayed Signal Cancellation (DSC) method, which has a similar working principle as the Fortescue matrix-based (FMB) filter, however, the motivation for the use of this technique has not been discussed. In [4], the authors provide a comparative approach between the SDBC and DSCC MMC topologies with regards to operation in unbalanced conditions. Their study used the Dual Second Order Generalized Integrator (DSOGI) for the control implementation of sequence extraction. Once again, the benefits of using specifically this method have not been highlighted.

Prior research on the SDBC MMC STATCOM does not focus on the sequence extraction method and does not state the reason for choosing one method over another. Additionally, these works do not highlight how the sequence extraction methods affect the control performances. The authors of this paper believe that each sequence extraction method has a certain delay and distinct functionalities which affect the behavior of the overall system differently. Based on that, the novelty of this work consists in analyzing SDBC MMC behavior while comparing four different sequence extraction methods: Decoupled Double Synchronous Reference Frame (DDSRF), DSOGI, Improved DSOGI (IDSOGI), and FMB filter. The aim is to show how each method would affect the system performances under both normal and abnormal conditions (voltage sag, Harmonics, DC offset). This analysis also helps to find the most fitting method for each grid scenario.

The SDBC-MMC control developed in this paper covers at once the positive and negative reactive power control as well as the harmonics control that can serve for future research.

The remainder of this paper is structured as follows. Section II gives a brief description of the system model and the adopted control strategies. Section III describes the four sequence extraction methods. Section IV tests and compares the performances of these methods under different grid conditions. Finally, Section V summarizes the findings of this study.

\section{SySTEM CONFIGURATION AND CONTROL}

\section{A. System Configuration}

The studied system is illustrated in Fig. 1. It consists of an SDBC MMC connected to a $20 \mathrm{kV}$ distribution power system through Maschinenfabrik Reinhausen platform, which comprises different connection transformers. In this work, the distribution system is represented by an equivalent three-phase voltage source $v_{e_{a, b, c}}$ in series with its equivalent impedance $L_{g}, R_{g}$. The SDBC MMC presents between each two phases of the grid voltage $u_{j}(j=12,23,31)$ a leg consisting of a reactance $L_{\Delta}$, and $N$ H-bridge cells.

A three-phase current source $i_{L_{a, b}, c}$ is connected in parallel with the MMC to emulate the nonlinear load and generate the current harmonics.

The variables $i_{x}(x=a b, b c, c a), i_{\Delta_{a, b, c}}, v_{m_{x}}, v_{c_{N}}$ and $i_{g_{a, b, c}}$ depicted in Fig. 1 refer to the internal currents, the compensating currents, the cluster voltages, the capacitors voltages, and the grid currents, respectively.

\section{B. MMC-SDBC STATCOM Control Strategy Under Distorted Grid Conditions}

The control strategy adopted for the MMC SDBC STATCOM is described in Fig. 2. It is divided into five main parts: Synchronization, decoupled Voltage Oriented Control (dVOC), Cluster Voltage Balancing Control, Harmonics Compensation/Rejection, and Voltage Reference Generation. Each 


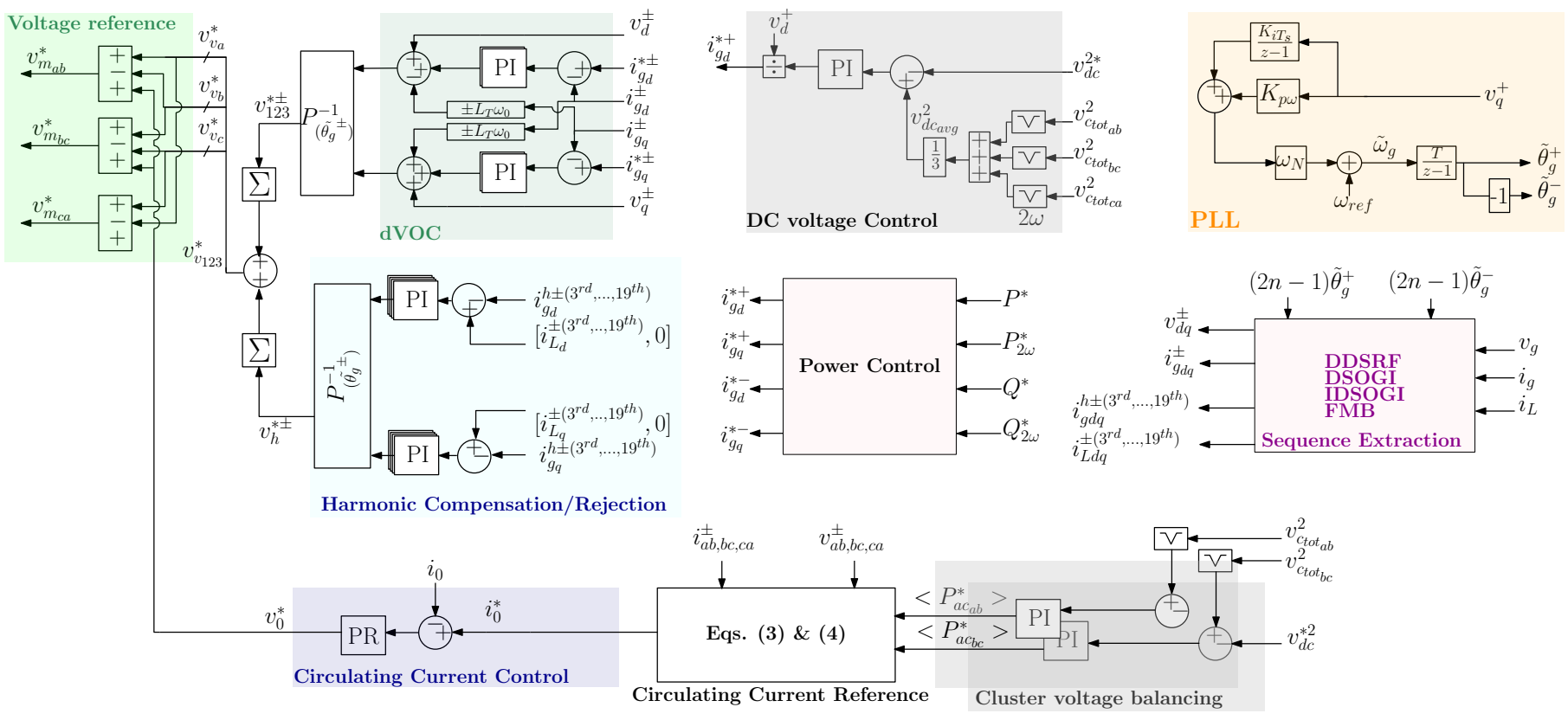

Fig. 2. MMC-SBDC STATCOM control strategy under distorted grid conditions

control function is highlighted in Fig. 2 and discussed in the following subsections.

1) Synchronization: A common method to achieve grid synchronization is using the Synchronous Reference Frame Phase-Locked Loop (SRF-PLL) [12], [21]. The SRF-PLL is used as part of the control system that aims to acquire the grid phase angle and the grid frequency [11], [12]. It aligns the rotating reference frame with the direct (d) positive sequence component of the voltage and sets the quadrature (q) positive sequence component to zero through a PI controller. Thus, $v_{d}^{+}$takes the value of the voltage magnitude, whereas $v_{q}^{+}$is used to detect the grid frequency and the positive phase angle [22], [23]. Since the negative sequence is rotating conversely to the positive sequence, the negative angle is determined by integrating $-\tilde{\omega}_{g}$.

2) Decoupled Voltage Oriented Control: The dVOC simultaneously manages the active current ( $d$-component) and the reactive current ( $q$-component) for both positive and negative sequences [11], [23], [24]. The $d-q$ currents are controlled via four-parallel PI controllers. $L_{T} \omega_{0} i_{\Delta_{d-q}}^{ \pm}$ terms and the grid-voltage feed-forwards are considered in the control feedback to decouple the $d-q$ components and to reject the grid disturbances, respectively. Note that $L_{T}$ is equal to the sum of $L_{\Delta} / 3$ and the equivalent inductance $L_{e q}$ of the transformer $(340 \mathrm{~V} / 4.3 \mathrm{kV})$.

$I_{\Delta_{d}}^{*+}$ and $I_{\Delta_{q}}^{*+}$ have the same functionality as $I_{\Delta_{d}}^{*}$ and $I_{\Delta_{q}}^{*}$ for the conventional current controller in balanced conditions, whereas $I_{\Delta_{d}}^{*-}$ and $I_{\Delta_{d}}^{*-}$ are regulated separately to compensate the current during unbalanced grid conditions. The references are provided by an outer DC voltage control and the power control [11], [24] given in (1).

The DC voltage in the DC control loop is defined as the average value of the total capacitor voltages $v_{c_{t o t x}}$. It contains fluctuations around the $\omega$ and $2 \omega$, which may affect the current controller operation. Therefore, a notch-filter or a moving average filter (MAF) are used in the DC voltage measurements to avoid the stated issue.

$$
\left[\begin{array}{c}
I_{g_{d}}^{+} \\
I_{g_{q}}^{+} \\
I_{g_{d}}^{-} \\
I_{g_{q}}^{-}
\end{array}\right]=\left[\begin{array}{cccc}
V_{d}^{+} & V_{q}^{+} & V_{d}^{-} & V_{q}^{-} \\
V_{d}^{-} & V_{q}^{-} & V_{d}^{+} & V_{q}^{+} \\
V_{q}^{-} & -V_{d}^{-} & -V_{q}^{+} & V_{d}^{+} \\
V_{q}^{+} & -V_{d}^{+} & V_{q}^{-} & -V_{d}^{-} \\
V_{q}^{-} & -V_{d}^{-} & -V_{q}^{+} & -V_{d}^{+} \\
V_{d}^{-} & -V_{q}^{-} & V_{d}^{+} & V_{q}^{+}
\end{array}\right]^{-1}\left[\begin{array}{c}
P^{*} \\
P_{c 2}^{*} \\
P_{s 2}^{*} \\
Q^{*} \\
Q_{c 2}^{*} \\
Q_{s 2}^{*}
\end{array}\right]
$$

When no grid faults occur, $I_{\Delta_{d}}^{-}$and $I_{\Delta_{q}}^{-}$are equal to zero. Contrariwise, during grid disturbances, two operation modes can be achieved by the STATCOM using the dual current controller. The negative sequence references $I_{\Delta_{d}}^{*-}$ and $I_{\Delta_{q}}^{*-}$ could be set to zero in order to eliminate the negative sequence and hence guarantee a symmetrical current, or, they can be used to suppress the second harmonic power oscillations by injecting an appropriate negative sequence current thanks to the power controller.

Note that either active or reactive power oscillations could be suppressed at a time. Therefore, either $P_{c 2}$ and $P_{s 2}$ are set to zero while $Q_{c 2}$ and $Q_{s 2}$ are omitted from the matrix or vice versa.

3) Cluster Voltage Balancing Control: The control of capacitors voltage balancing across each leg depends on the total power flow between the STATCOM and the grid. Note that under unbalanced conditions, the negative sequence components of both voltage and current contribute to the total power exchange between the converter and the AC grid. This may cause unbalanced power sharing among legs if no adequate control is adapted. Referring to [4], the circulating current could be employed as a degree of freedom to ensure capacitors voltage balancing under balanced and distorted grid conditions. As illustrated in Fig. 2, the cluster voltage balancing 
control calculates the reference value of the circulating current, which can be written as:

$$
i_{0}=\hat{I}_{0} \cos \left(\tilde{\theta_{g}}+\phi_{0}\right)
$$

The square values of the cluster voltages are controlled via two $P I$-controllers. The power disturbances in the clusters are denoted by $\left(P_{a b}^{*}, P_{b c}^{*}, P_{c a}^{*}\right)$. According to [4], the magnitude and the phase of the circulating current reference are given by:

$$
\begin{gathered}
I_{0}=\frac{P_{a b}^{*}-X_{11}}{X_{1} \cos \phi_{0}+X_{2} \sin \phi_{0}} \\
\phi_{0}=\arctan \frac{\left(P_{b c}^{*}-X_{22}\right) X_{1}-\left(P_{a b}^{*}-X_{11}\right) X_{3}}{\left(P_{a b}^{*}-X_{11}\right) X_{4}-\left(P_{b c}^{*}-X_{22}\right) X_{2}}
\end{gathered}
$$

with,

$$
\begin{gathered}
X_{11}=\frac{\hat{V}^{+} \hat{I_{\Delta}^{-}}}{2} \cos \left(\delta^{+}-\phi^{-}+\frac{\pi}{3}\right) \\
+\frac{\hat{V}^{-} I_{\delta}^{+}}{2} \cos \left(\delta^{-}-\phi^{+}-\frac{\pi}{3}\right) \\
X_{22}=\frac{\hat{V}^{+} \hat{I_{\Delta}^{-}}}{2} \cos \left(\delta^{+}-\phi^{-}+\pi\right) \\
+\frac{\hat{V}^{-} \hat{I_{\Delta}^{+}}}{2} \cos \left(\delta^{-}-\phi^{+}-\pi\right) \\
X_{1}=\frac{\sqrt{3}}{2} \hat{V}^{+} \cos \left(\delta^{+}+\frac{\pi}{6}\right)+\frac{\sqrt{3}}{2} \hat{V}^{-} \cos \left(\delta^{-}-\frac{\pi}{6}\right) \\
X_{2}=\frac{\sqrt{3}}{2} \hat{V}^{+} \sin \left(\delta^{+}+\frac{\pi}{6}\right)+\frac{\sqrt{3}}{2} \hat{V}^{-} \sin \left(\delta^{-}-\frac{\pi}{6}\right) \\
X_{3}=\frac{\sqrt{3}}{2} \hat{V}^{+} \cos \left(\delta^{+}-\frac{\pi}{2}\right)+\frac{\sqrt{3}}{2} \hat{V}^{-} \cos \left(\delta^{-}+\frac{\pi}{2}\right) \\
X_{4}=\frac{\sqrt{3}}{2} \hat{V}^{+} \sin \left(\delta^{+}-\frac{\pi}{2}\right)+\frac{\sqrt{3}}{2} \hat{V}^{-} \sin \left(\delta^{-}+\frac{\pi}{2}\right)
\end{gathered}
$$

In this paper, the generated circulating current reference is compared to the measured one and controlled through a Proportional Multi-Resonant controller (PMR) given in (5), instead of the proportional controller conventionally used in the literature. The aim is to perfectly track the reference signal, which improves the quality of generated output voltage $v_{m}^{*}$.

$$
H_{P R}=k_{p}+\sum T_{s} \frac{1-z^{-1} \cos \left(\omega_{0} T_{s}\right)}{1-2 z^{-1} \cos \left(\omega_{0} T_{s}\right)+z^{-2}}
$$

The PR is discretized using the Impulse Variant Method (IVM) because of its stability with high range of harmonics.

4) Harmonics Compensation/Rejection: The harmonics control concept is illustrated in Fig. 2 and given with more details in [8]. Usually, the odd harmonics are targeted as they are more dominant in the grid. The measured current at the PCC $\left(i_{g}\right)$ undergoes sequence extraction at different phase angles/harmonic frequencies (i.e. $3^{\text {rd }}, 5^{\text {th }}, \ldots$ ), thus transforming each harmonic component to its DC equivalent represented in the $d-q$ frame. The transformed DC signals are compared with the corresponding reference signal. If the reference is set to zero, then harmonic compensation takes place and the STATCOM takes all the harmonics from the grid. Otherwise, the harmonics remain on the grid side and the STATCOM output is unaffected. Similar to the dVOC, each harmonic component is regulated via dual-PI controllers.
5) Voltage Reference Generation: The aim of this control is to generate a voltage reference for each leg that will be used thereafter to generate the modulation index $m$ in the case of an average model, or the pulses $g_{i}$ in the case of a detailed switching model [25], [26]. In this paper, only the average model is used since the paper focuses on the system level. The reference voltage of each leg is determined based on the reference voltages $v_{v_{1,2,3}}^{*}$ generated by the current controllers and $v_{0}$ generated by the circulating current controller, such that:

$$
\left[\begin{array}{c}
v_{m_{a b}}^{*} \\
v_{m_{b c}}^{*} \\
v_{m_{c a}}^{*}
\end{array}\right]=\left[\begin{array}{l}
v_{v_{1}}^{*}-v_{v_{2}}^{*}+v_{0}^{*} \\
v_{v_{2}}^{*}-v_{v_{3}}^{*}+v_{0}^{*} \\
v_{v_{3}}^{*}-v_{v_{1}}^{*}+v_{0}^{*}
\end{array}\right]
$$

\section{SEQUENCE EXTRACTION}

Under non-ideal grid conditions, the MMC may fail to accurately synchronize with the AC grid and/or inject the desired current due to the interaction between the positive and negative sequences [22]. By implementing two Park's transformations using two frames rotating in opposite directions $(+\theta,-\theta)$, the resultant is two signals formed by the coupling of both positive and negative sequences [22]. These coupled signals are given by (7) and (8), respectively. $x$ in the equations represents any electrical variable and the bar on top (e.g. $\overline{x_{d}^{+}}$) represents a DC component of this variable.

$$
\begin{aligned}
& {\left[\begin{array}{l}
x_{d}^{+} \\
x_{q}^{+}
\end{array}\right]=\left[\begin{array}{l}
\overline{x_{d}^{+}} \\
\overline{x_{q}^{+}}
\end{array}\right]+\left[\begin{array}{cc}
\cos (2 \theta) & \sin (2 \theta) \\
-\sin (2 \theta) & \cos (2 \theta)
\end{array}\right]\left[\begin{array}{l}
\overline{x_{d}^{-}} \\
x_{q}^{-}
\end{array}\right]} \\
& {\left[\begin{array}{l}
x_{d}^{-} \\
x_{q}^{-}
\end{array}\right]=\left[\begin{array}{l}
\overline{x_{d}^{-}} \\
\overline{x_{q}^{-}}
\end{array}\right]+\left[\begin{array}{cc}
\cos (-2 \theta) & \sin (-2 \theta) \\
-\sin (-2 \theta) & \cos (-2 \theta)
\end{array}\right]\left[\begin{array}{l}
\overline{x_{d}^{+}} \\
x_{q}^{+}
\end{array}\right]}
\end{aligned}
$$

To remove this coupling effect, a proper method of sequence extraction must be utilized. Four sequence extraction methods are investigated in this section.

\section{A. Decoupled Double Synchronous Reference Frame (DDSRF)}

DDSRF was presented in [13] as a solution for accurately detecting the positive sequence components under distorted or unbalanced voltage conditions. This method utilizes two rotating synchronous reference frames and decouples the sequence interaction by subtracting the interference of each sequence on the other. (9) and (10) provide a clear demonstration of the working principle of the DDSRF, which are obtained by rearranging (7) and (8). The implementation of (9) and (10) can be seen in Fig. 3. Following [13], the extracted components from the DDSRF go through a low-pass filter (LPF) that is tuned to a cut-off frequency of $\tilde{\omega}_{g} / \sqrt{2}$.

$$
\begin{aligned}
& {\left[\begin{array}{l}
\overline{x_{d}^{+}} \\
x_{q}^{+}
\end{array}\right]=\left[\begin{array}{l}
x_{d}^{+} \\
x_{q}^{+}
\end{array}\right]-\left[\begin{array}{cc}
\cos (2 \theta) & \sin (2 \theta) \\
-\sin (2 \theta) & \cos (2 \theta)
\end{array}\right]\left[\begin{array}{l}
\overline{x_{d}^{-}} \\
x_{q}^{-}
\end{array}\right]} \\
& {\left[\begin{array}{l}
\overline{x_{d}^{-}} \\
x_{q}^{-}
\end{array}\right]=\left[\begin{array}{l}
x_{d}^{-} \\
x_{q}^{-}
\end{array}\right]-\left[\begin{array}{cc}
\cos (-2 \theta) & \sin (-2 \theta) \\
-\sin (-2 \theta) & \cos (-2 \theta)
\end{array}\right]\left[\begin{array}{l}
\overline{x_{d}^{+}} \\
\overline{x_{q}^{+}}
\end{array}\right]}
\end{aligned}
$$




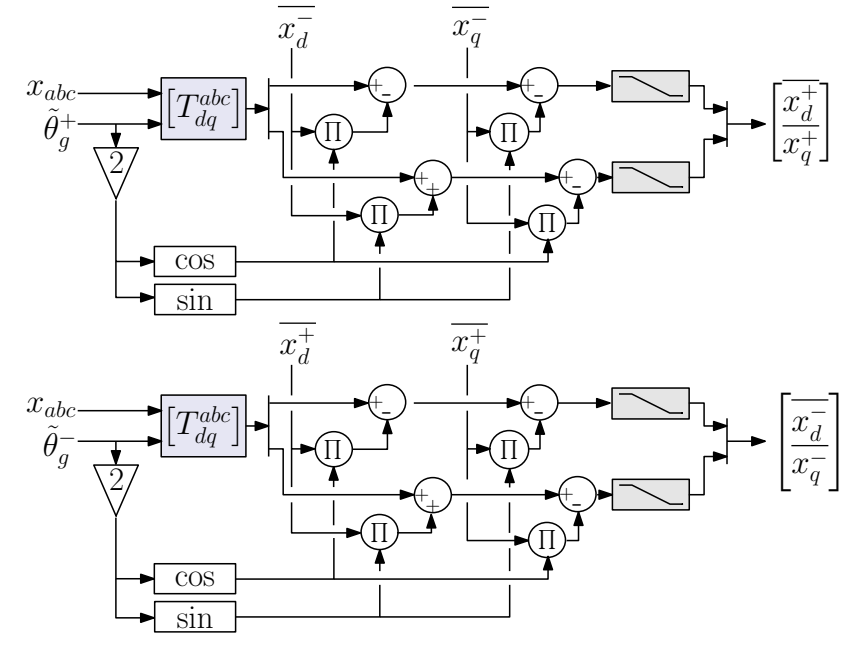

Fig. 3. Sequence extraction using DDSRF

\section{B. Dual Second Order Generalized Integrator (DSOGI)}

SOGI is the most popular method used for grid synchronization [27], [28] and is made up of a frequency adaptive bandpass filter. One of the main features of the SOGI is its dual outputs: the (band-pass) filtered signal $x_{f}$ and a $90^{\circ}$ shifted orthogonal (low-pass) filtered signal $x_{f}^{q}$. The SOGI filter is described by the block diagram in Fig. 4, where, $\mathrm{k}$ is the constant responsible for changing the bandwidth of the filter.

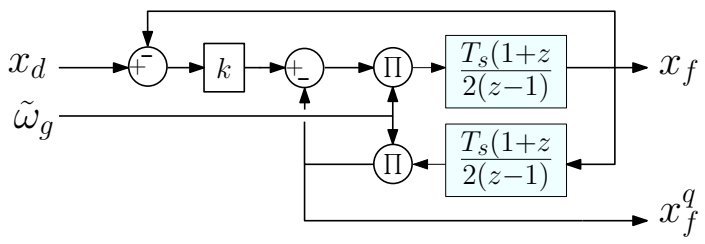

Fig. 4. SOGI filter

Following [29], and using the Fortescue matrix, the positive and negative sequences can be calculated using (11) and (12) respectively. DSOGI is a combination of two SOGIs implemented for $\alpha$ and $\beta$ components. The implementation of the sequence extraction using DSOGI is represented in Fig. 5.

$$
\begin{aligned}
& {\left[\begin{array}{l}
x_{\alpha}^{+} \\
x_{\beta}^{+}
\end{array}\right]=\frac{1}{2}\left[\begin{array}{cc}
1 & -e^{-j(\pi / 2)} \\
e^{-j(\pi / 2)} & 1
\end{array}\right]\left[\begin{array}{l}
x_{\alpha} \\
x_{\beta}
\end{array}\right]} \\
& {\left[\begin{array}{l}
x_{\alpha}^{-} \\
x_{\beta}^{-}
\end{array}\right]=\frac{1}{2}\left[\begin{array}{cc}
1 & e^{-j(\pi / 2)} \\
-e^{-j(\pi / 2)} & 1
\end{array}\right]\left[\begin{array}{l}
x_{\alpha} \\
x_{\beta}
\end{array}\right]}
\end{aligned}
$$

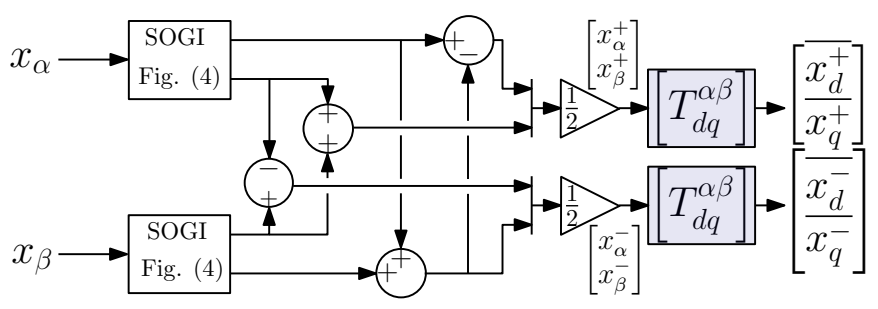

Fig. 5. Sequence extraction using DSOGI
C. Improved Dual Second Order Generalized Integrator (IDSOGI)

Since the $x_{f}$ and $x_{f}^{q}$ signals of the SOGI are only filtered input signals, the SOGI has no ability to suppress DC offsets [27]. On the other hand, controlling the gain $k$ to attenuate low-order harmonics will come at the expense of the system dynamics [27]. Therefore, authors in [6] have proposed an improved SOGI (ISOGI) with the aim of tackling the limitations of the conventional one i.e., compensating DC offsets and increasing the control accuracy without compromising the system dynamics. Its block diagram is presented in Fig. 6 .

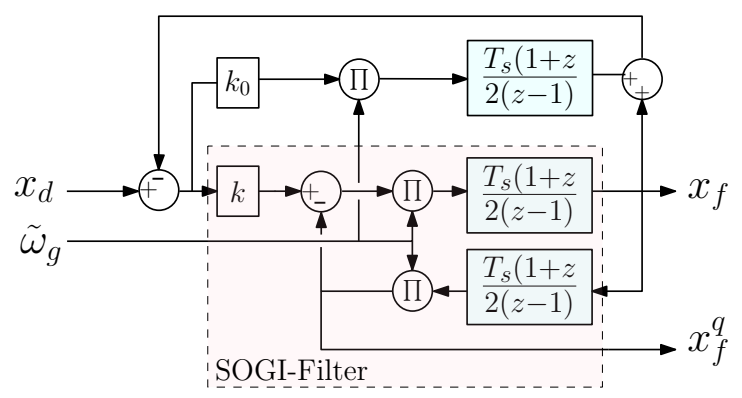

Fig. 6. ISOGI filter

This method increases the filtering capabilities of the SOGI by transforming it to a third order transfer function as shown in Fig. 6. The additional integrator is used to generate a state variable that is equal to the DC offset, which is subtracted from the input signal. The parameter $k_{0}$ is a gain used in the DC offset estimation channel, and it is tuned based on the roots of the system closed-loop transfer function [6]. Similar to the DSOGI method, the sequences are extracted by substituting the conventional SOGI filter from Fig. 5 by ISOGI and therefore forming the IDSOGI.

\section{Fortescue Matrix-Based Filter in $\alpha \beta$ Frame (FMB filter)}

This technique decouples the sequences in the $\alpha \beta$ frame following (11) and (12). It uses an LPF to remove second order harmonics and a High Pass Filter (HPF) to shift the signal by $90^{\circ}$. Both are set with a cut-off frequency of 100 Hz. The block diagram of this method is shown in Fig. 7.

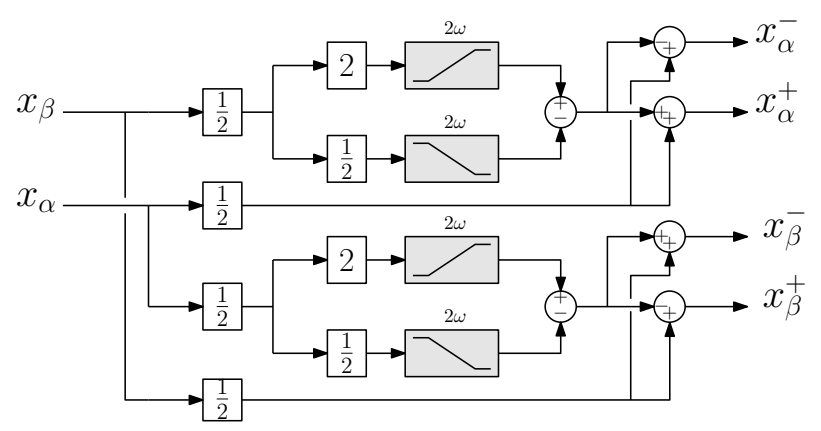

Fig. 7. Sequence extraction using fortescue matrix-based filter in $\alpha \beta$ frame 


\section{Verification AND COMPARISON OF THE SEQUENCE EXTRACTION METHODS}

To verify the proficiency of the proposed control based on the aforementioned sequence extraction methods, timedomain simulations were performed in Maltab/Simulink and conducted with the system and control parameters listed in Table I. The simulation time step is $T_{\text {sim }}=1 u s$. The system is running with $T_{s y s}=T_{s i m}$ and the control is running with $T_{s}=50 u s$.

Four test cases are performed in the following subsections to assess the system behavior based on each sequence extraction method: 1) Reactive power change, 2) Single-phase voltage sag, 3) Harmonics injection, 4) Single-phase grid voltage measurement DC offset.

Note that the tuning of the control loops is unchanged when switching from one method to the other and from one test case to the other. This is important to find out the most suitable method that can deal with different distorted conditions without a need for additional control adaptations.

TABLE I

SYSTEM AND CONTROL PARAMETERS

\begin{tabular}{||c|c|c||}
\hline Parameters & Value & Units \\
\hline \hline Grid phase-to-phase RMS Voltage $\left(V_{e}\right)$ & $5.27 \mathrm{e} 3$ & $\mathrm{kV}$ \\
Nominal Output Power & $0.36 \mathrm{e} 6$ & $\mathrm{VA}$ \\
Fundamental Frequency & 50 & $\mathrm{~Hz}$ \\
Grid Resistance $\left(R_{g}\right)$ & 0.1 & $\mathrm{Ohms}$ \\
Grid Inductance $\left(L_{g}\right)$ & 0.001 & $\mathrm{H}$ \\
Number of Submodule Cells per Leg & 21 & - \\
Capacitance per Submodule $\left(C_{S M}\right)$ & $10 \mathrm{e}-3$ & $\mathrm{~F}$ \\
STATCOM Leg Resistance $\left(R_{\Delta}\right)$ & 0.0082 & $\mathrm{p} . \mathrm{u}$ \\
STATCOM Leg Reactance $\left(L_{\Delta}\right)$ & 0.082 & $\mathrm{p} . \mathrm{u}$ \\
Equivalent Transformer Impedance $340 \mathrm{~V} / 4.3 \mathrm{kV} X_{e}$ & 0.08 & $\mathrm{p} . \mathrm{u}$ \\
Equivalent Transformer Impedance $20 \mathrm{kV} / 400 \mathrm{~V} X_{e}$ & 0.04 & $\mathrm{p} . \mathrm{u}$ \\
Current Controller Integral Gain $\left(K_{i}^{ \pm}\right)$ & 0.5416 & $\mathrm{p} . \mathrm{u}$ \\
Current Controller Proportional Gain $\left(K_{p}^{ \pm}\right)$ & 1.3540 & $\mathrm{p} . \mathrm{u}$ \\
DC voltage $T_{R}^{5 \%}$ & 200 & $\mathrm{~ms}$ \\
Voltage balancing control $T_{R}^{5 \%}$ & 400 & $\mathrm{~ms}$ \\
Circulating current $T_{R}^{5 \%}$ & 5 & $\mathrm{~ms}$ \\
PLL $T_{R}^{5 \%}$ & 100 & $\mathrm{~ms}$ \\
Voltage FF time-constant LPF $\tau$ & 4 & $\mathrm{~ms}$ \\
Harmonics Current Controller Integral Gain $\left(K_{i}^{h}\right)$ & 0.5416 & $\mathrm{p} . \mathrm{u}$ \\
Harmonics Current Controller Propor. Gain $\left(K_{p}^{h}\right)$ & 1.3540 & $\mathrm{p} . \mathrm{u}$ \\
SOGI and ISOGI gains $k, k_{0}$ & 1 & $\mathrm{p} . \mathrm{u}$ \\
\hline \multicolumn{2}{|c}{}
\end{tabular}

\section{A. Case A: Reactive Power Change}

A step change of $Q=0.9$ p.u is applied to the system at $t=$ $0.5 \mathrm{~s}$. The grid current and the reactive power responses based on each extraction method are presented in Fig. 9. The results show a stable system operation with the four techniques. However, their transient behavior is different, i.e., the FMB filter presents the lowest overshoot of $46 \%$ compared to the DDSRF, DSOGI, and IDSOGI which exhibited an overshoot of $156 \%, 178.5 \%$, and $178 \%$, respectively. Moreover, with the FMB filter, the system recovers stably its equilibrium point within less than $15 \mathrm{~ms}$. With DDSRF, DSOGI, and IDSOGI, the system took approximately $50 \mathrm{~ms}$ to recover. This difference is explained by the fact that DDSRF, DSOGI, and IDSOGI introduce $\tau \geq 4.5 \mathrm{~ms}$ time-delay in the current and voltage measurements and thereby a delay in the control,
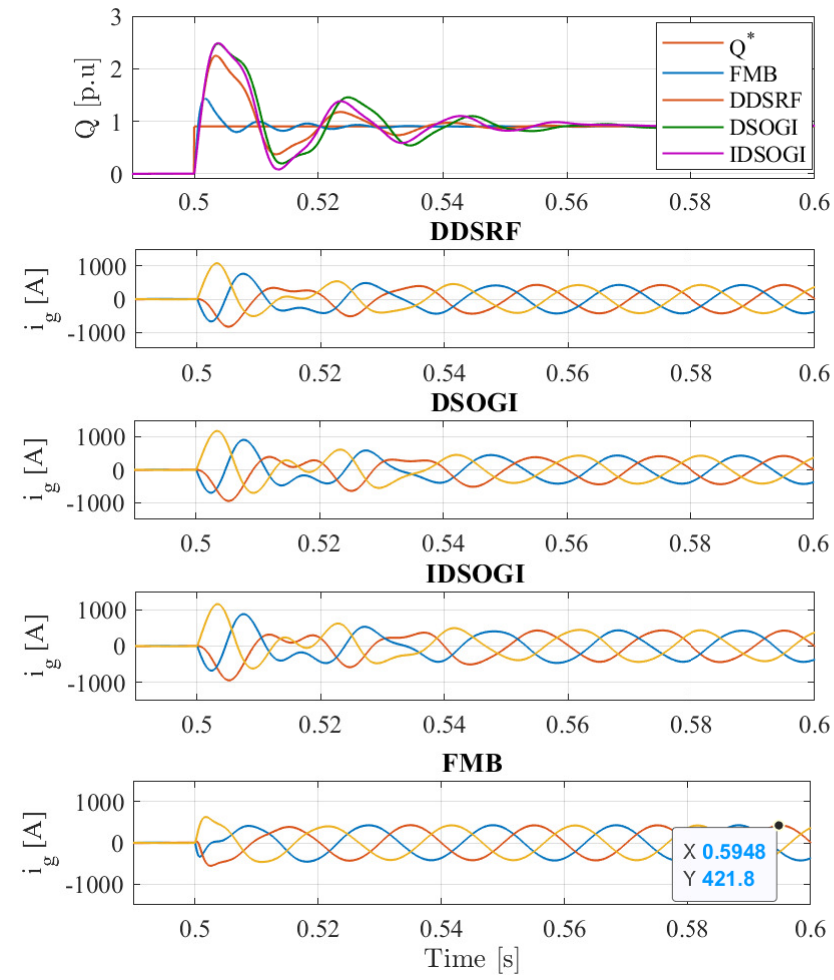

Fig. 8. Grid current dynamics with respect to 0.9 p.u reactive power change
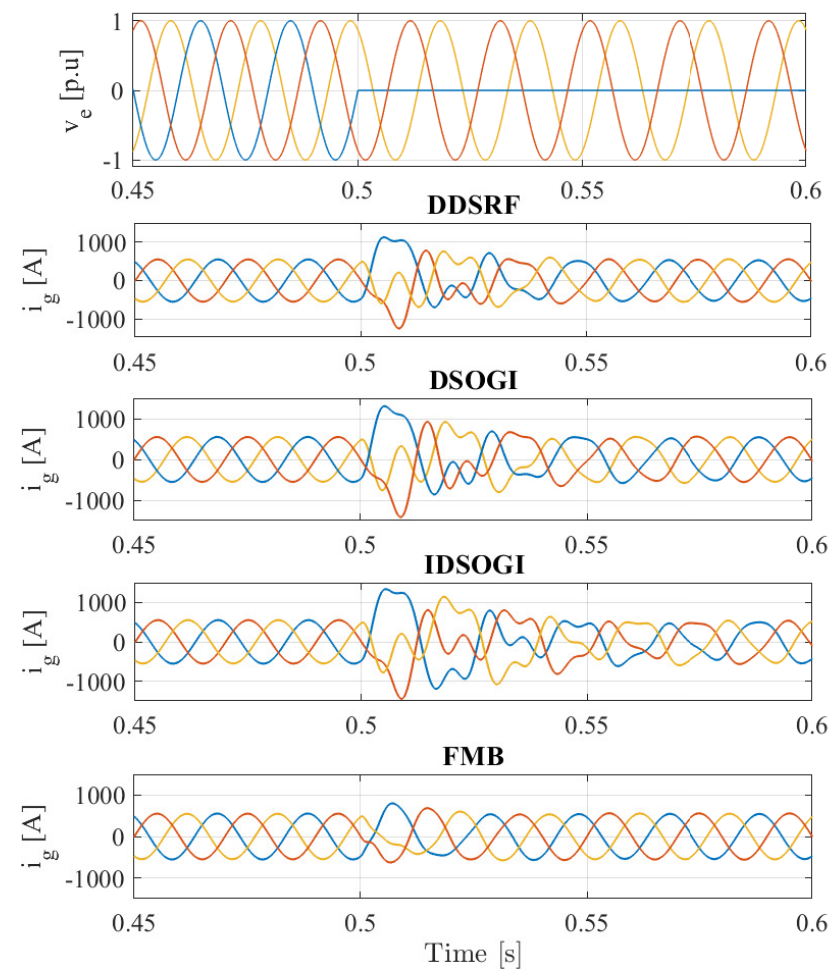

Fig. 9. Grid current dynamics under single-phase $100 \%$ voltage sag

whereas, the FMB filter introduces a smaller time-delay of $\tau=1.6 \mathrm{~ms}$, which results in faster regulation. Based on the obtained results, the FMB filter presented the best dynamic performances in case of an operating point change under balanced grid conditions. 


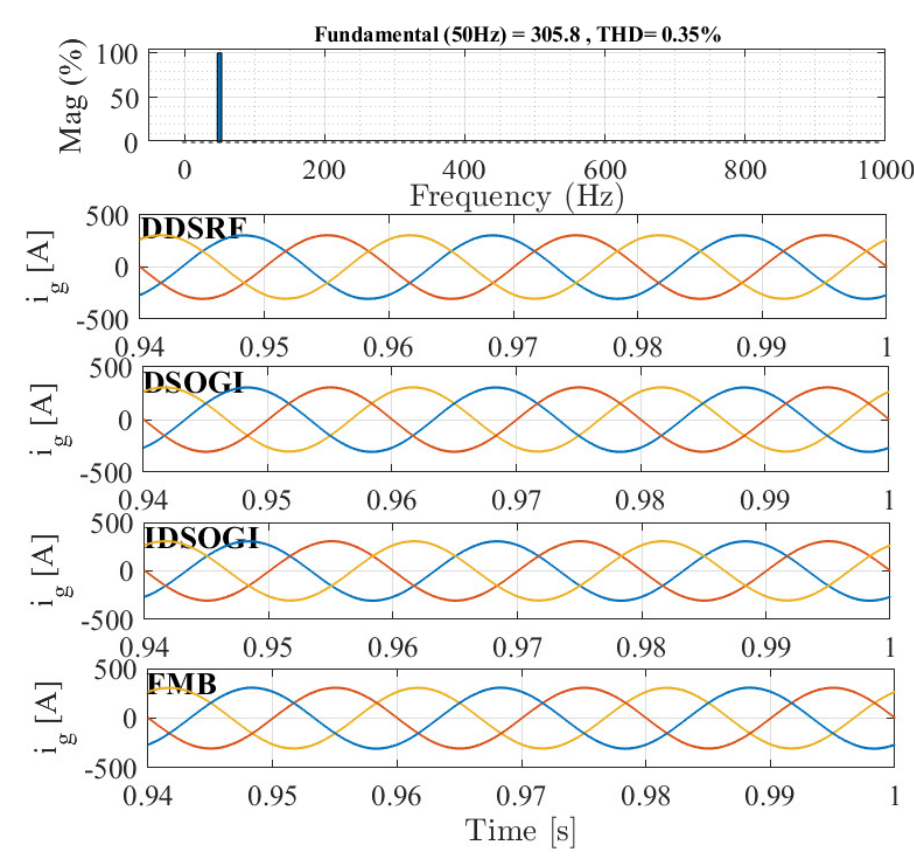

Fig. 10. Harmonics compensation

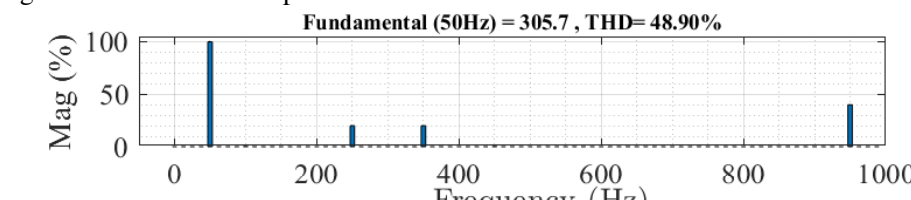

年

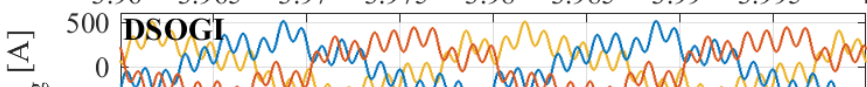

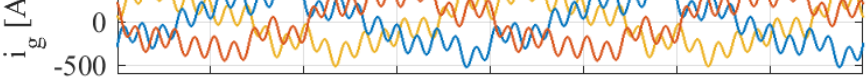

$\begin{array}{lllllllll}3.96 & 3.965 & 3.97 & 3.975 & 3.98 & 3.985 & 3.99 & 3.995 & 4\end{array}$

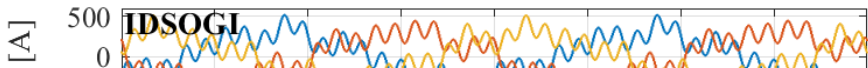

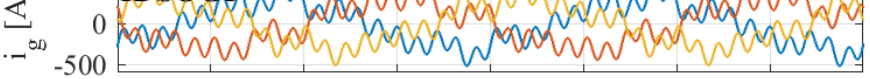

$\begin{array}{lllllllll}3.96 & 3.965 & 3.97 & 3.975 & 3.98 & 3.985 & 3.99 & 3.995 & 4\end{array}$

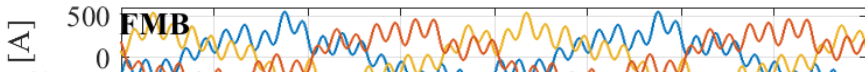

$\begin{array}{lllllllll}3.96 & 3.965 & 3.97 & 3.975 & 3.98 & 3.985 & 3.99 & 3.995 & 4\end{array}$ Time $[\mathrm{s}]$

Fig. 11. Harmonics rejection

\section{B. Case B: A Single-Phase Voltage Sag}

The voltage sag along with the grid currents for each method is given in Fig. 12. For this test case, the system is initially operating with $Q=1$ p.u, then a $100 \%$ single phase voltage sag occurs at $t=1 \mathrm{~s}$. The results globally show the proficiency of the dVOC adopted to compensate the negative sequence of the currents caused by the voltage unbalance. Compared to test case A, the voltage sag leads to the same overcurrent transient peaks for each method. However, regarding the recovery time duration, the system based on the FMB filter stabilized within less than $20 \mathrm{~ms}$, whereas, the DDSRF, DSOGI, and IDSOGI
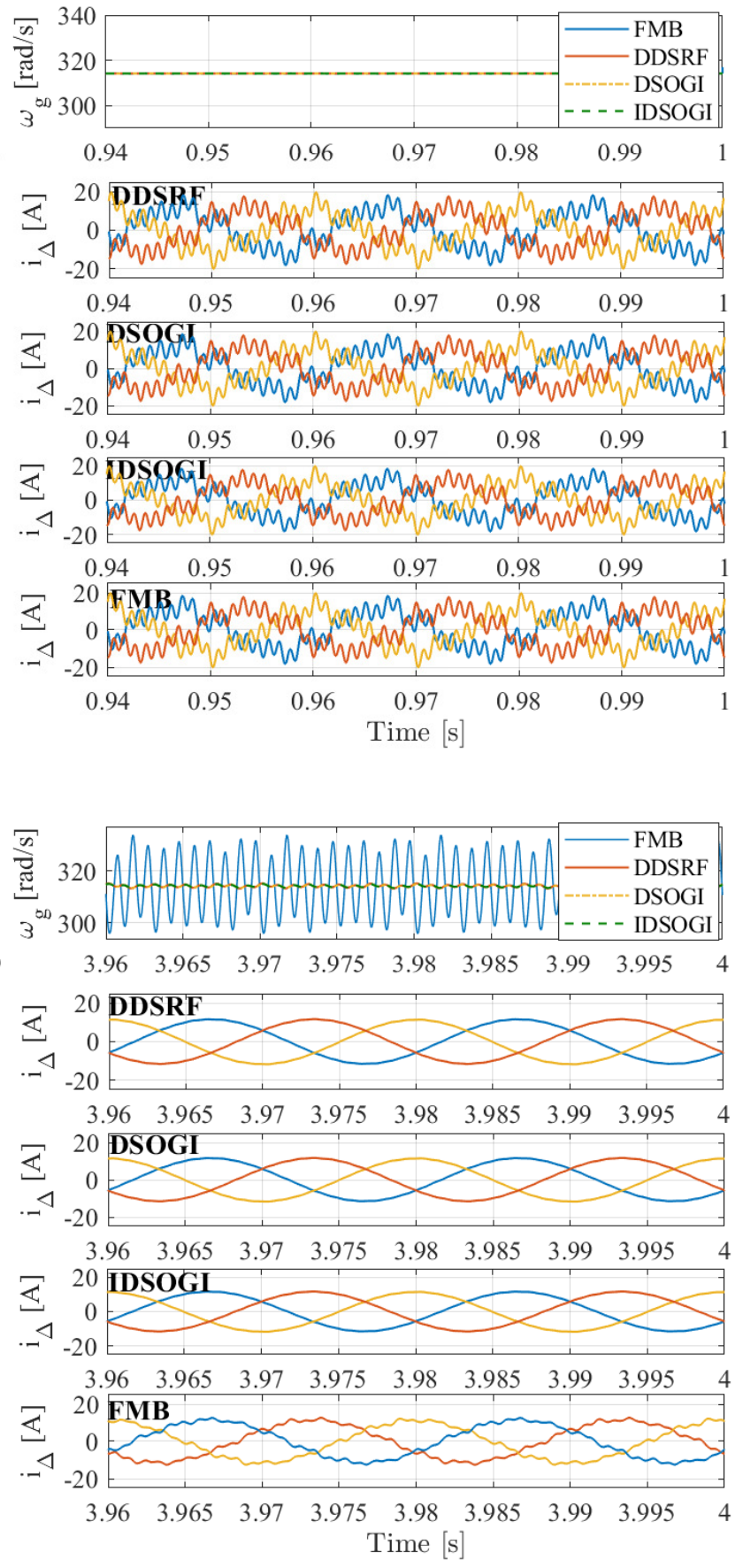

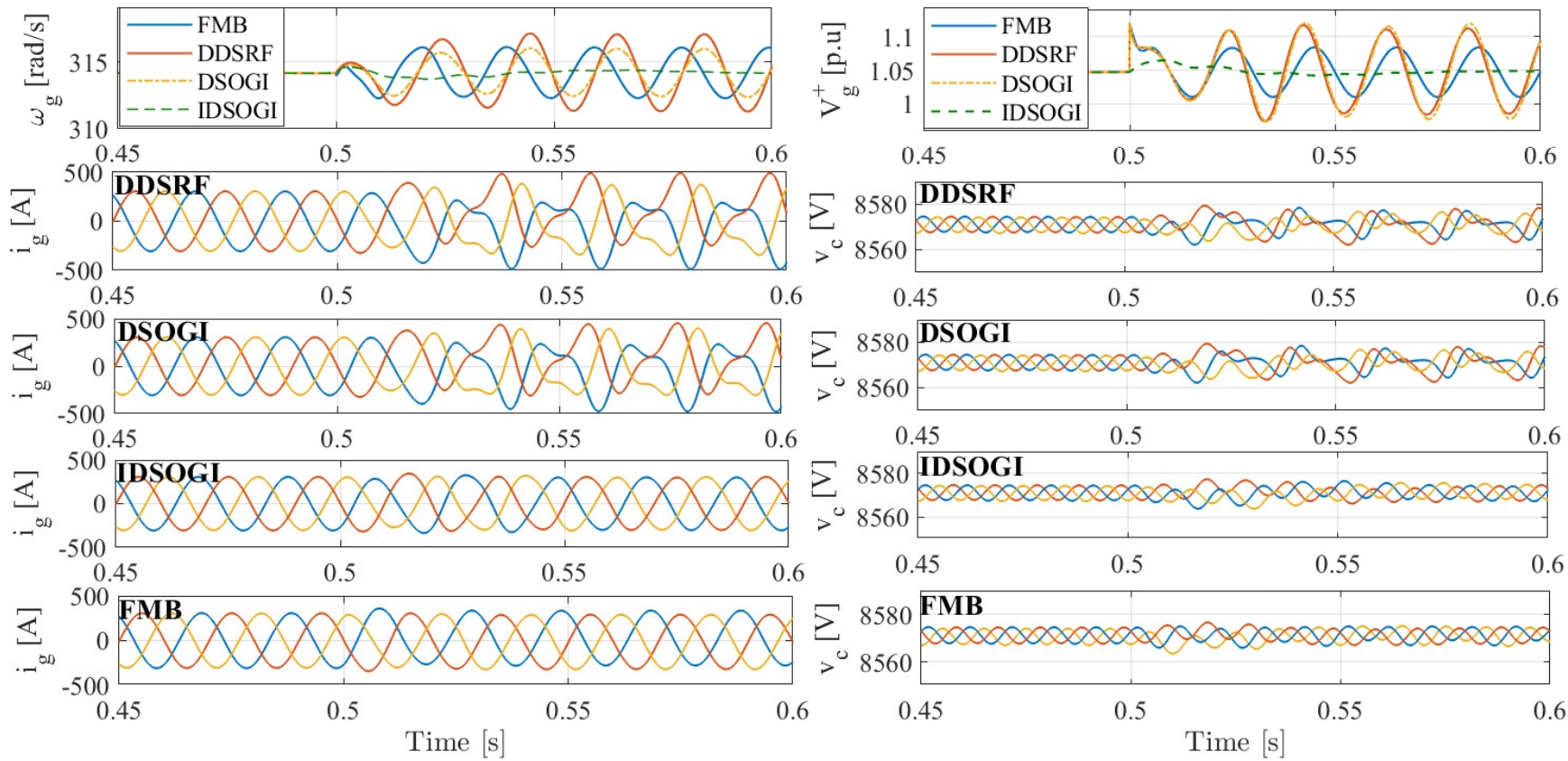

Fig. 12. System behavior under AC voltage measurement Single-Phase DC Offset of $10 \%$

operating with $Q^{*}=0.5$ p.u, then, three odd harmonics were injected to the grid, the $5^{t h}, 7^{\text {th }}$ and $19^{\text {th }}$ harmonics with the magnitudes 0.1 p.u, 0.1 p.u, and 0.2 p.u, respectively. Both compensation and rejection modes are evaluated. Simulation results are gathered in Fig. 10 and Fig. 11, where, the grid current $i_{g}$, the MMC compensated current $i_{\Delta}$, the Total Harmonic Distortion (THD), and the estimated grid frequency $\tilde{\omega}_{g}$ are displayed.

1) Control Mode I: Harmonic Compensation: One can notice from the obtained results in Fig. 10 that the STATCOM absorbed the harmonic content as excepted, while the grid current is kept free from any harmonics (THD $=0.35 \%)$. The harmonic content of the grid current is almost the same with the four studied methods. Therefore, the DDSRF, DSOGI, IDSOGI, and FMB filter could be interchangeable in such a condition.

2) Control Mode II: Harmonic Rejection: The results for this control mode can be seen in Fig. 11. Conversely to the harmonics compensation mode, the STATCOM output currents $i_{\Delta}$ show almost no harmonic content with the DDSRF, DSOGI, and IDSOGI since the harmonics are rejected from the STATCOM output and completely fed by the AC grid (THD $=48.9 \%)$. This is not completely the case with the FMB filter, actually, the grid current harmonics naturally affect the AC voltage $v_{g}$ used by the PLL for synchronization. And since the FMB has no harmonics filtering capability as the DDSRF, DSOGI, and IDSOGI, the estimated frequency through it is consequently affected in steady state, which results in an inaccurate harmonics rejection. In this control mode, the four extraction methods guarantee a stable operation, nevertheless, the DDSRF, DSOGI, and IDSOGI show higher performances against the FMB filter.

\section{Case D: AC voltage measurement Single-Phase DC Offset}

A 0.1 p.u DC offset is applied to the measured AC voltage $v_{g}$ phase A at $t=0.5 \mathrm{~s}$. The effect of the DC offset on the grid voltage magnitude positive sequence $V_{g}^{+}$, the estimated grid frequency $\tilde{\omega}_{g}$, the cell's capacitor voltages $v_{c}$ is analyzed. The results are shown in Fig. 12. The DC offset commonly introduces a fundamental frequency oscillation on the $\mathrm{AC}$ voltage. Out of the four sequence extraction methods, only the IDSOGI is able to reject the DC offset, and consequently ensures the system requirements by injecting a balanced $\mathrm{AC}$ current. From the other side, the DSOGI, the DDSRF, and the FMB filter are affected by the DC offset leading to the migration of the grid voltage oscillations to the estimated grid frequency. Despite that the three methods are impacted by this event, the consequences are significantly different i.e., with the DDSRF and the DSOGI, the grid currents and capacitor voltages are deteriorated and are out of control. Whereas, with the FMB, the capacitor voltages are almost non-affected and the grid currents are the image of the $\mathrm{AC}$ voltage containing the same DC offset degree of $10 \%$, but still having a sinusoidal waveform.

\section{CONCLUSION}

In this paper, four sequence extraction methods based on a decoupled control algorithm for the SDBC-MMC STATCOM have been analyzed under different grid conditions and are compared in terms of stability, dynamic performances, and functionality. Regarding stability aspects, the four extraction methods resulted in stable operation of the studied system in various grid conditions. The main differences between the extraction methods are noted when comparing their effect on the dynamic performances and their functionalities. The difference in the transient response of the system is mainly 
attributed to the time delay introduced in the measurements by each method. On the basis of the simulation results, the Fortescue Matrix-Based filter is the most suitable technique to be used for the SDBC-MMC STATCOM to achieve higher transient dynamic performances under normal and unbalanced grid conditions due to its short time-delay. In terms of functionality, the filtering capabilities of each method, especially in case of different harmonics and DC offsets, were assessed. The IDSOGI showed the most promising results as compared to its alternatives (DDSRF and DSOGI) as it has better filtering capabilities against harmonics and DC offsets. Since, the main goals in a power system are to support the AC grid, reject the grid disturbances, and guarantee the overall system stability, IDSOGI presented the best compromise between dynamic performances and functionalities for these targets. For future work, the authors suggest a combination of the FMB filter and the IDSOGI as a part of the control of the SDBCMMC STATCOM. Such that, the FMB filter would be used to extract the symmetrical components of the current due to its fast response time and rejection of $2 \omega$ oscillations, while the IDSOGI could be used in the voltage measurements to guarantee the grid harmonics and DC offsets rejection utilizing its superior filtering capabilities.

\section{REFERENCES}

[1] T. Qoria, Q. Cossart, C. Li, X. Guillaud, F. Colas, F. Gruson, and X. Kestelyn, "WP3-Control and Operation of a Grid with 100\% ConverterBased Devices. Deliverable 3.2: Local control and simulation tools for large transmission systems," MIGRATE Project, Tech. Rep., 2018.

[2] F. Shahnazian, E. Adabi, J. Adabi, E. Pouresmaeil, K. Rouzbehi, E.M.G. Rodrigues, J.P.S. Catalão, "Control of MMC-Based STATCOM as an Effective Interface between Energy Sources and the Power Grid," Electronics, 2019; 8(11):1264.

[3] H. Akagi, "Classification, terminology, and application of the modular multilevel cascade converter (MMCC)," The 2010 International Power Electronics Conference - ECCE ASIA -, 2010, pp. 508-515,

[4] A. F. Cupertino, J. V. M. Farias, H. A. Pereira, S. I. Seleme and R. Teodorescu, "Comparison of DSCC and SDBC Modular Multilevel Converters for STATCOM Application During Negative Sequence Compensation," in IEEE Transactions on Industrial Electronics, vol. 66, no. 3, pp. 23022312, March 2019 ,

[5] P. Wu, Y. Chen and P. Cheng, "The Delta-Connected Cascaded HBridge Converter Application in Distributed Energy Resources and Fault Ride Through Capability Analysis," in IEEE Transactions on Industry Applications, vol. 53, no. 5, pp. 4665-4672, Sept.-Oct. 2017, doi: 10.1109/TIA.2017.2702110.

[6] J. Li, J. Zhao, J. Wu and P. Xu, "Improved dual second-order generalized integrator PLL for grid synchronization under non-ideal grid voltages including DC offset," 2014 IEEE Energy Conversion Congress and Exposition (ECCE), 2014, pp. 136-141, doi: 10.1109/ECCE.2014.6953386.

[7] S. Lubura, S.-A. Lale, M. `Ika, and M. Šoja, "Single-phase phase locked loop with DC offset and noise rejection for photovoltaic inverters," IET Power Electron., vol. 7, no. 9, pp. 2288-2299, Sep. 2014.

[8] F. Blaabjerg, R. Teodorescu, M. Liserre and A. V. Timbus, "Overview of Control and Grid Synchronization for Distributed Power Generation Systems," in IEEE Transactions on Industrial Electronics, vol. 53, no. 5 , pp. 1398-1409, Oct. 2006, doi: 10.1109/TIE.2006.881997.

[9] O. J. K. Oghorada and L. Zhang, "Unbalanced and Reactive Load Compensation Using MMCC-Based SATCOMs With Third-Harmonic Injection," in IEEE Transactions on Industrial Electronics, vol. 66, no. 4, pp. 2891-2902, April 2019, doi: 10.1109/TIE.2018.2849962.

[10] G. Buticchi, E. Lorenzani and G. Franceschini, "A DC Offset Current Compensation Strategy in Transformerless Grid-Connected Power Converters," in IEEE Transactions on Power Delivery, vol. 26, no. 4, pp. 2743-2751, Oct. 2011, doi: 10.1109/TPWRD.2011.2167160.

[11] E. Ozsoy et al., "Control Strategy for a Grid-Connected Inverter under Unbalanced Network Conditions-A Disturbance Observer-Based Decoupled Current Approach", Energies, vol. 10, no. 7, p. 1067, 2017. doi: $10.3390 /$ en 10071067
[12] K. Sharifabadi, L. Harnefors, H.-P. Nee, R. Teodorescu, and S. Norrga, "Design, Control and Application of Modular Multilevel Converters for HVDC Transmission Systems", 1st ed.: John Wiley Sons, 2018.

[13] P. Rodriguez, J. Pou, J. Bergas, J. I. Candela, R. P. Burgos and D. Boroyevich, "Decoupled Double Synchronous Reference Frame PLL for Power Converters Control," in IEEE Transactions on Power Electronics, vol. 22, no. 2, pp. 584-592, March 2007, doi: 10.1109/TPEL.2006.890000.

[14] A. Meligy, T. Qoria, and I. Colak, "SDBC-MMC STATCOM Control Under Unbalanced Grid Conditions Based on Different Sequence Extraction Methods", IECON 2021 The 47th Annual Conference of the IEEE Industrial Electronics Society, 2021.

[15] T. Hao, F. Gao, and T. Xu, "Fast Extraction of Symmetrical Components from Distorted Three-Phase Signals Based on Asynchronous-Rotational Reference Frame," Journal of Power Electronics, vol. 19, no. 4, pp. 1045-1053, Jul. 2019.

[16] H. Ahmed and M. Benbouzid, "Adaptive Observer-Based GridSynchronization and Sequence Extraction Techniques for Renewable Energy Systems: A Comparative Analysis”, Applied Sciences, vol. 11, no. 2 , p. 653 , 2021, doi: 10.3390/app11020653.

[17] D. Basic, M. Geske and S. Schroeder, "Limitations of the H-bridge multilevel STATCOMs in compensation of current imbalance," 2015 17th European Conference on Power Electronics and Applications (EPE'15 ECCE-Europe), 2015, pp. 1-10, doi: 10.1109/EPE.2015.7311687.

[18] P. Wu, H. Chen, Y. Chang and P. Cheng, "Delta-connected cascaded H-bridge converter application in unbalanced load compensation," 2015 IEEE Energy Conversion Congress and Exposition (ECCE), 2015, pp. 6043-6050, doi: 10.1109/ECCE.2015.7310507.

[19] J. Jung, J. Lee, S. Sul, G. T. Son and Y. Chung, ”DC Capacitor Voltage Balancing Control for Delta-Connected Cascaded H-Bridge STATCOM Considering Unbalanced Grid and Load Conditions," in IEEE Transactions on Power Electronics, vol. 33, no. 6, pp. 4726-4735, June 2018, doi: 10.1109/TPEL.2017.2730244.

[20] E. Behrouzian, M. Bongiorno and H. Z. De La Parra, "Investigation of negative sequence injection capability in H-bridge multilevel STATCOM," 2014 16th European Conference on Power Electronics and Applications, 2014, pp. 1-10, doi: 10.1109/EPE.2014.6910883.

[21] H. Ahmed, M. Benbouzid, M. Ahsan, A. Albarbar and M. Shahjalal, "Frequency Adaptive Parameter Estimation of Unbalanced and Distorted Power Grid," in IEEE Access, vol. 8, pp. 8512-8519, 2020, doi: 10.1109/ACCESS.2020.2964058.

[22] F. Sevilmiş and H. Karaca, "Performance analysis of SRF-PLL and DDSRF-PLL algorithms for grid interactive inverters", International Advanced Researches and Engineering Journal, pp. 116-122, 2019. Available: $10.35860 /$ iarej.412250.

[23] D. Siemaszko and A. Rufery, "Double-Frame Current Control with a Multivariable PI Controller and Power Compensation for Weak Unbalanced Networks", pp.97-113, 2015. Available: 10.5170/CERN-2015003.97

[24] Hong-Seok Song and Kwanghee Nam, "Dual current control scheme for PWM converter under unbalanced input voltage conditions," in IEEE Transactions on Industrial Electronics, vol. 46, no. 5, pp. 953-959, Oct. 1999, doi: 10.1109/41.793344.

[25] S. Kannan, C. Poongothai, I. Colak and W. Ali, "Selective Harmonic Elimination for Modular Multilevel Converter with Averaging and Circulating Current Control," 2018 7th International Conference on Renewable Energy Research and Applications (ICRERA), 2018, pp. 221-226,

[26] A. Bissal, W. Ali, R. Leedham, M. Snook, I. Elsabrouty and I. Colak, "A Hybrid Pulse Width Modulation Technique with Temperature Control for Modular Multilevel Converters," 2020 22nd European Conference on Power Electronics and Applications (EPE'20 ECCE Europe), 2020, pp. P.1-P.9,

[27] J. Xu, H. Qian, Y. Hu, S. Bian and S. Xie, "Overview of SOGIBased Single-Phase Phase-Locked Loops for Grid Synchronization Under Complex Grid Conditions," in IEEE Access, vol. 9, pp. 39275-39291, 2021, doi: 10.1109/ACCESS.2021.3063774

[28] H. Ahmed and M. Benbouzid, "Simplified second-order generalized integrator-frequency-locked loop," Advances in Electrical and Electronic Engineering, vol. 17, no. 4, pp. 405-412, 2019.

[29] P. Rodríguez, A. Luna, R. S. Muñoz-Aguilar, I. Etxeberria-Otadui, R. Teodorescu and F. Blaabjerg, "A Stationary Reference Frame Grid Synchronization System for Three-Phase Grid-Connected Power Converters Under Adverse Grid Conditions," in IEEE Transactions on Power Electronics, vol. 27, no. 1, pp. 99-112, Jan. 2012, doi: 10.1109/TPEL.2011.2159242. 